\title{
Performance Analysis for Uniform and Binomial Distribution on Contention Window using DSRC and Wi-Fi Direct Standard
}

\author{
Nurain Izzati Shuhaimi****, Heriansyah*, Tutun Juhana*, Adit Kurniawan* \\ * Sekolah Teknik Elektro dan Informatika, Institut Teknologi Bandung, J1. Ganesa No.10 Bandung, Indonesia \\ ** Fakulti Kejuruteraan Elektrik, Universiti Teknologi MARA, 40450 Shah Alam, Malaysia
}

\section{Article Info}

Article history:

Received Jul 18, 2015

Revised Aug 28, 2015

Accepted Sep 13, 2015

Keyword:

Binomial algorithm

DSRC

Uniform and non-uniformly

Distributed backoff timer

VANET

Wi-Fi direct

\begin{abstract}
In this paper, we present a mathematical analysis on the performance and behavior of uniformly distributed and non-uniformly distributed backoff timer based on binomial algorithm by using two standards which is the conventional DSRC and the latest Wi-Fi Direct. DSRC is a well-known technology being considered as the most promising wireless standard in VANET. On the other hand, as the latest wireless networking standard, the potential of Wi-Fi Direct technology should be concerned. We evaluates these standards using uniform and binomial distribution for contention window under mathematical modelling in order to analyze the average throughput and collision probability performance. The results show that binomial distribution in Wi-Fi Direct standard is $7.05 \%$ and $97.13 \%$ better than uniform distribution, in terms of average throughput and collision probability, respectively. Thus we can defer that Wi-Fi Direct is feasible to be used as an alternative standard since it has been considered as potential competitor of DSRC in VANET.
\end{abstract}

Copyright $@ 2015$ Institute of Advanced Engineering and Science. All rights reserved.

\section{Corresponding Author:}

Nurain Izzati Shuhaimi,

Sekolah Teknik Elektro dan Informatika,

Institut Teknologi Bandung,

Jl. Ganesa No. 10 Bandung, Indonesia.

Email: nurainizzati_shuhaimi@yahoo.com

\section{INTRODUCTION}

In recent years, a growing number of mobile applications has been introduced through broad adoption of wireless network. Particularly in vehicular communication system, vehicle can acts as a mobile node that able to send data to the neighbouring vehicle or roadside unit, and vice versa. A vehicular ad-hoc network (VANET) consists of vehicle-to-vehicle and vehicle-to-infrastructure communication based on local area network technology. The transmission of information on vehicular node includes a variety of services ranging from road safety, traffic efficiency and infotainment applications [1]. In terms of standard, IEEE 802.11p or also known as WAVE has been defined as the communication protocols of physical and medium access control (MAC) layer for vehicular environments. The operating frequency is fixed in Dedicated Short Range Communication (DSRC) band spanning from 5.85 to $5.925 \mathrm{GHz}$, in which seven channels are allocated where 1 channel specified as control channel $(\mathrm{CCH})$ while the remaining 6 channels are set as service channels (SCHs) [2].

In accordance with the initial design of 802.11 wireless networking standards, $802.11 \mathrm{p}$ allows vehicular nodes to access the medium by using Carrier Sense Multiple Access with Collision Avoidance (CSMA/CA) [3] which implemented by Enhanced Distribution Coordination Function (EDCF) that based on DCF. With EDCF mechanism, a vehicular node is allowed to start packet transmission only if the channel is idle for Distributed Inter Frame Space (DIFS) duration before the backoff procedure is permitted to resume. A transmitting station must make sure that the medium is idle for this required duration before attempt to do 
the transmitting process [4]. Since there is a possibility of stations choosing the same backoff number, they may send the data in same slot which leads to probability of colliding with each other. That is why a uniformly distributed random backoff number is designed in order to alleviate this probability of collision.

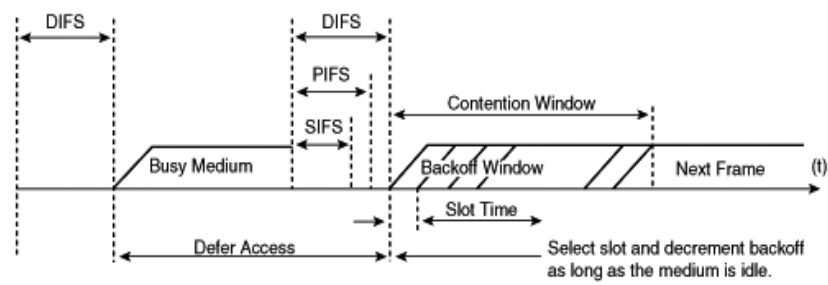

Figure 1. Basic access method

As in Figure 1, after sensing that the medium is busy, then the station shall defer for another free DIFS time and selects a uniform random backoff number from contention window (CW). Usually the station will doubles the current $\mathrm{CW}$ so that it can selects another backoff until reaching the maximum size of $\mathrm{CW}$ [5]. The equation of the backoff period is determined by:

$$
C W=2^{n-1} * C W_{\min } \text { where } n \text { is number of retry }
$$

However, the main characteristics in vehicular environment in which nodes typically move on a road with higher mobility and frequent network topology change dynamically than those in other ad-hoc network scenarios lead to some issues and challenges in network design, especially on MAC layer [6]. Aiming to improve the MAC performance in VANET, we propose a mathematical analysis by implementing a non-uniformly distributed backoff timer based on binomial distribution for contention window which can reduce the collision probability while increase the performance of throughput. Apart from using the conventional DSRC as communication protocol of physical and MAC layer for vehicular environment, we will use Wi-Fi Direct standard as an alternate. Although we know that DRSC provides a reliable communication between vehicle-to-vehicle, problem concerning on the cost of DSRC hardware become a constraint. Because of the fact that DSRC requires a dedicated hardware, we can assume that it cannot be considered as the best solution to warn pedestrians or cyclists, since it may require them to carry DSRC equipment all the time [7], [8]. Because of this shortcoming, we choose Wi-Fi Direct standard as an alternative for vehicle-to-vehicle communication since it has been considered as potential competitor other than DSRC standard.

The remainder of this paper is divided into the following sections, where Section 2 discusses the overview of related works, Section 3 describes on mathematical analysis, and Section 4 presents on result and discussion. While the conclusion and future work is given in Section 5 and 6, respectively.

\section{RELATED WORKS}

A lot of studies have been done that substantially aiming to improve the MAC performance in vehicular networks such as by modifying the conventional Binary Exponential Backoff (BEB) algorithm. In these studies, they made a proposal whether to adjust the initial value of $\mathrm{CW}$ based on the estimated density vehicles or modify the method of selecting backoff integer with a deterministic function, $f$ instead of a random variable technique. For instance, the authors in [9], [10] explained that when the network congestion becomes severe, the size of contention window will depends strongly on the number of competing vehicles alongside with the number of transmission retries. While in [11], the authors made a proposal by applying the scheme of collision alleviation. Here, instead of adjusting the contention window size according to the number of competing nodes, they proposed the adjusting of contention window size according to the average number of retries.

Otherwise, the authors in [12] proposed a simple modification on BEB algorithm which can begin with a relatively large value for contention window, after that divide it by two each time when a real-time safety message on the control channel expires, particularly focused the delivery of safety messages. Per contra, the value for large contention window in their enhanced protocol remains undefined. Also, a group of researchers have come out with one-to-one function mapping from several unique reference to a slot number in contention window in order to increase the chances of successful reception probability on the control channel. For a vehicular node, the unique reference in this approach is relevant to its own geographical location [13], while the study by [14] is on its MAC address of wireless interface. As in [15], the nodes 
granted to choose in advance a free backoff value for the next packet waiting for transmission to the updated backoff by applying Transmit and Reserve (TAR) channel access scheme. Also, the nodes need to piggyback the chosen backoff value in current packet that ready to transmit. Without considering the increasing number of vehicular nodes, the range of TAR cycle can rising rapidly which leads into unbounded delay.

In a nutshell, we can conclude that size of contention window is the major factor affecting the MAC performance in vehicular network. Thus in this research, we propose a modified backoff algorithm that can decrease the possibility of continuous collisions among the competing vehicles by generating a nonuniformly distributed backoff timer based on binomial distribution.

\section{MATHEMATICAL ANALYSIS}

Referring to well-known Bianchi model for modelling the CSMA/CA mechanism presented in [16], we calculate the throughput and probability of a packet transmission failure due to collision in both DSRC and Wi-Fi Direct standard. In conventional uniform distribution, the throughput, $\tau$ which is the average information transmitted in a slot time over the average duration of a slot time can be calculated as follows:

$$
\tau=\frac{E[\text { Payload in formation transmitted in a slot time }}{\begin{array}{c}
E[\text { Duration of slot time }] \\
P_{S} \times D
\end{array}}
$$

Where $D$ is the average packet payload size, $P_{i}=(1-p)^{n}$ is the probability that a slot is idle, $P_{S}=$ $\frac{n p(1-p)^{n-1}}{1-(1-p)^{n}}$ is the probability that a slot transmits data successfully, and $P_{c}=1-(1-p)^{n-1}$ is the probability that a slot is in a collision state. The transmission probability, $p$ can be calculated with minimum value of contention window as $p=\frac{2}{c W_{\text {min }}+1}$. Further, $t_{\text {slot }}$ refer to idle slot time, $t_{\text {success }}$ is the time spent to transmit a packet successfully and $t_{c o l}$ is the time spent during packet collision.

As for binomial distribution function, the normalized throughput is the same as in Equation (2) but the difference will be on the calculation for probability of idle, success and collision. Noted that probability mass function for binomial distribution [17] is given by:

$$
f(x: p, n)=\left(\begin{array}{l}
n \\
x
\end{array}\right) p^{x}(1-p)^{n-x}
$$

Where $n$ is number of trial, $x$ is number of success in $n$ trial and $p$ is probability of success in single trial. Thus based on Equation 3, we can determined $P_{i}=(1-p)^{n}$ as probability that the slot is in idle state, $P_{s}=(n p)(1-p)^{n-1}$ is the probability that a transmission occurring on the channel is successful, and $P_{c}=1-\left[(1-p+n p)(1-p)^{n-1}\right]$ is the probability of collision transmission.

\section{RESULT AND ANALYSIS}

We performed an analysis for both uniform and binomial distribution using the parameters for DSRC and Wi-Fi Direct standard as summarized in Table 1, in order to analyse the performance of throughput and probability of collision. For the analysis, we decided to use CSMA/CA mechanism protocol for transmitting instead of RTS/CTS for two reasons. First, this protocol wastes bandwidth due to long waiting period and frequently exchanging control packets. Secondly, RTS/CTS will causes more complicated and additive interference scenarios due to collisions among control and data messages [18]. Further, we fixed the contention window at 31 timeslots, as it is the value used in almost papers in the literature while the contending nodes are varied from 1 to 20 .

Table 1. Simulation parameter

\begin{tabular}{ccc}
\hline Parameter & DSRC & Wi-Fi Direct \\
\hline Data rate & $6 \mathrm{Mbps}$ & $6 \mathrm{Mbps}$ \\
ACK & $192 \mathrm{bits}$ & $192 \mathrm{bits}$ \\
DIFS & $58 \mu \mathrm{s}$ & $34 \mu \mathrm{s}$ \\
SIFS & $32 \mu \mathrm{s}$ & $16 \mu \mathrm{s}$ \\
Propagation delay & $2 \mu \mathrm{s}$ & $1 \mu \mathrm{s}$ \\
MAC header & $256 \mathrm{bits}$ & $256 \mathrm{bits}$ \\
PHY header & $192 \mathrm{bits}$ & $192 \mathrm{bits}$ \\
Slot time & $13 \mu \mathrm{s}$ & $9 \mu \mathrm{s}$ \\
\hline
\end{tabular}


From Figure 2, we can observe that the average throughput of uniform distribution in Wi-Fi Direct standard is better than DSRC standard by $39.2 \%$. Here, we can say that by using uniform distribution, the probability of idle and success in both standards seems to reduce as the number of nodes are increasing, resulting on the degradation of average throughput. In terms of standard, Wi-Fi Direct has a better performance since time taken of $t_{\text {slot }}, t_{\text {success }}$ and $t_{c o l}$ recorded much lower value which will causes a greater throughput. It follows the theoretical study in previous study in which the throughput is inversely proportional to the average duration of a slot time [19].

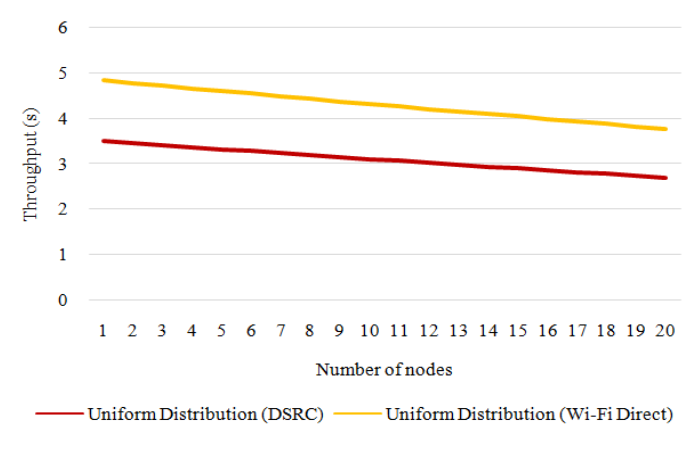

Figure 2. Throughput for different nodes using uniform distribution

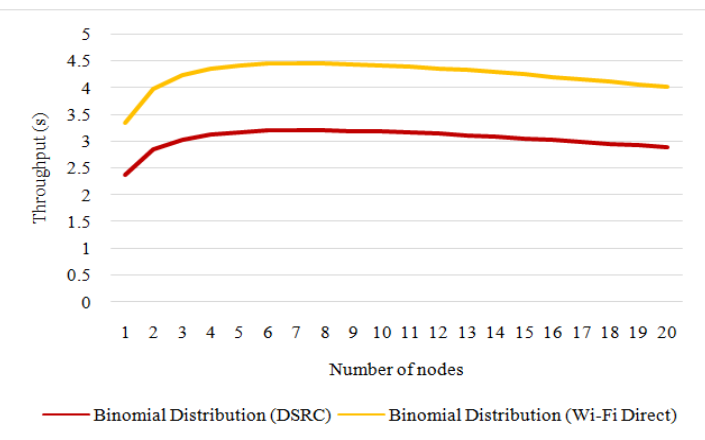

Figure 3. Throughput for different nodes using binomial distribution

While in Figure 3, we can see that the average throughput of binomial distribution in Wi-Fi Direct standard is better than DSRC standard by $39.13 \%$. As we can see from the graph, when the contending nodes are varied from 1 to 7 nodes, the average throughput is lower particularly when the nodes are small. Conversely, when the contending nodes are varied until 20 nodes, the throughput using both standards seems to be stable and gradually reduced. Here, we can observe that by using binomial distribution, the probability of success in both standards seems to reduce as the number of competing nodes are small. On the contrary, the probability of success getting higher as the number of nodes are increasing. Therefore from the result, we can summarize that binomial distribution is suitable to use compared to uniform distribution in heavy load traffic condition.

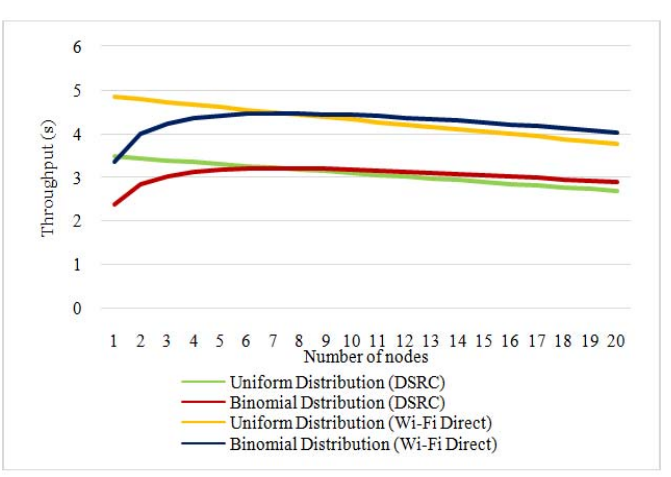

Figure 4. Throughput for different nodes using both distribution

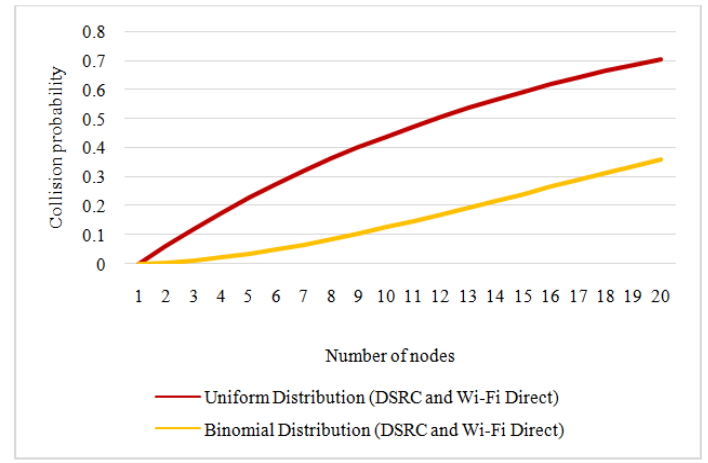

Figure 5. Collision probability for different nodes using both distribution

Figure 4 shows the comparison of throughput efficiency between both uniform and binomial distribution in DSRC and Wi-Fi Direct standard. Here, we can conclude that Wi-Fi Direct can works better than DSRC standard, especially by generating a non-uniformly distributed backoff timer based on binomial distribution, where binomial distribution in Wi-Fi Direct is $7.05 \%$ better than uniform distribution in Wi-Fi Direct. From the result, we can see that binomial distribution having slightly better performance than uniform distribution. This is due to the transmission data rate that we set at $6 \mathrm{Mbps}$ for both standards. It is known that Wi-Fi Direct offers faster data rate over two-way area coverage, which is up to $250 \mathrm{Mbps}$ compared to

Performance Analysis for Uniform and Binomial Distribution on Contention... (Nurain Izzati Shuhaimi) 
DSRC that offers only 6-27 Mbps. In this study, we choose the minimum data rate at $6 \mathrm{Mbps}$ in order to see the fair difference between both standards. We also can summarize that Wi-Fi Direct can have a good throughput at high contending nodes only if we set the transmission data rate at higher number, which is up to $250 \mathrm{Mbps}$.

Afterward, Figure 5 shows the collision probability between both uniform and binomial distribution in DSRC and Wi-Fi Direct standard. Here, we can observe that binomial distribution in both standards is $97.23 \%$ better than uniform distribution. We can infer that when the number of nodes increase, the collision probability will also increase. Noticeably the collision probability will always grow up with the increasing in number of data flows. Further, the collision probability of binomial distribution is much lower than uniform distribution because nodes can select their backoff integer in order to avoid on choosing the same backoff integer as the other nodes do in conventional scheme. It means, each node can enter different channels, resulting on reducing the possibility of collision. Thus we can conclude that performance of binomial distribution on contention window performs better than uniform distribution in both DSRC and Wi-Fi Direct standard.

\section{CONCLUSION}

This paper presented the analysis of two different distribution which is uniform distribution and non-uniformly distributed backoff timer based on binomial algorithm for contention window by using DSRC and Wi-Fi Direct standard. In a nutshell, the simulation results show that binomial distribution outperforms the conventional uniform distribution in terms of average throughput and collision probability. Noted that the explanation on these results have been presented as in previous section. By looking at both standards, we can conclude that Wi-Fi Direct has a better performance than DSRC since it shows that it has a good achievement. For a conclusion, we know that DSRC has been considered as the most promising wireless standard but there are several circumstances that should be emphasized. Instead of relying on this conventional standard that has been widely used in vehicular network, the extra advantage of using Wi-Fi Direct where it is known as a peer-to-peer standard that allows Wi-Fi devices, can connect directly among each other without the need for a wireless hotspot. Thus we can say that Wi-Fi Direct as the latest wireless networking standard can overtake DSRC performance in the near future.

For future work, we will evaluate and study more on the behaviour of binomial backoff algorithm, in addition we can test the protocol performance with real large scale of test bed experiment using NS-2.

\section{REFERENCES}

[1] N. M. Sadek, et al., "A Robust Multi-RAT VANET/LTE for Mixed Control and Entertainment Traffic," Journal of Transportation Technologies, Vol. 5, pp. 113-121, 2015.

[2] K. A. Hafeez, et al., "Optimizing the Control Channel Interval of DSRC for Vehicular Safety Applications," Globecom Workshop on Wireless Networking and Control for Unmanned Autonomous Vehicles, pp. 1481-1486, 2014.

[3] V. D. Khairnar1 and K. Kotecha, "Performance of Vehicle-to-Vehicle Communication using IEEE 802.11p in Vehicular Ad-hoc Network Environment," International Journal of Network Security and Applications (IJNSA), Vol. 5, No. 2, 2013.

[4] G. Shanti, et al., "Performance Analysis of IEEE 802.11e under Binomial Backoff Algorithm," IEEE International Advance Computing Conference (IACC), pp. 267-270, 2014.

[5] V. Tiwari, "Performance Enhancement in VANET with Admission Control and Contention Window Adjustment," Master of Applied Science Thesis, University of Victoria, Canada, 2012.

[6] W. Alasmary and W. Zhuang, "The Mobility Impact in IEEE 802.11p Infrastructure less Vehicular Networks," Elsevier Ad Hoc Networks Journal, vol. 10, no. 2, pp. 222-230, 2012.

[7] C. Satish, "Inter Vehicular Communication for Collision Avoidance using Wi-Fi Direct," Master Thesis, Rochester Institute of Technology, New York United States, 2014.

[8] A. Priyanka, "Increased Persistence of Wi-Fi Direct Networks for Smartphone-based Collision Avoidance," Master Thesis, Rochester Institute of Technology, New York United States, 2014.

[9] C. Chrysostomou, et al., "Applying Adaptive QoS-aware Medium Access Control in Priority Based Vehicular Ad Hoc Networks," Proceeding on 16 $6^{\text {th }}$ IEEE Symposium on Computers and Communications, pp. 741-747, 2011.

[10] R. Reinders, et al., "Contention Window Analysis for Beaconing in VANETs," Proceeding on $7^{\text {th }}$ International Wireless Communication and Mobile Computing Conference, pp. 1481-1487, 2011.

[11] D. J. Deng, et al., "A Collision Alleviation Scheme for IEEE 802.11p VANETs," Springer Wireless Personal Communications, Vol. 56, No. 3, pp. 371-383, 2011.

[12] R. Stanica, et al., "Enhancements of IEEE 802.11p Protocol for Access Control on VANET Control Channel," IEEE International Conference of Communication, pp. 1-5, 2011.

[13] A. Ahmad, et al., "A New Adapted Back-off Scheme for Broadcasting on IEEE 1609.4 Control Channel in VANET," Proceeding on Ad Hoc Networking Workshop, pp. 9-15, 2012. 
[14] K.J. Song, et al., "Distributed Periodic Access Scheme (DPAS) for the Periodic Safety Messages in the IEEE 802.11p WAVE," $3^{\text {rd }}$ Proceeding International Conference on Communications and Mobile Computing, pp. 465468, 2011.

[15] I. Khoufi, et al., "TAR Channel Access Mechanism for VANET Safety-Critical Situations," Proceeding on Intelligent and Advanced System, 2012.

[16] G. Bianchi, "Performance Analysis of the IEEE 802.11 Distributed Coordination Function," Journal on Selected Areas in Communications, pp. 535-547, 2000.

[17] J. Wroughton and T. Cole, "Distinguishing between Binomial, Hypergeometric and Negative Binomial Distributions," Journal of Statistics Education, Vol. 21, No. 1, 2013.

[18] C. Liu, "An Innovative MultiVariable Control Framework for Effective Wireless Resource Management," $P h D$ Thesis, University of Alberta, Canada, 2012.

[19] J. Sheng, "Performance Modelling of IEEE 802.11 Ad Hoc Networks under Time-Varying Channel," PhD Thesis, Rensselaer Polytechnic Institute Troy, New York, 2008.

\section{BIBLIOGRAPHIES OF AUTHORS}

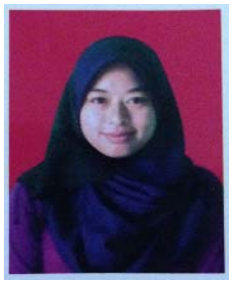

Nurain Izzati Shuhaimi is a $\mathrm{PhD}$ candidate in Telecommunication Engineering at Bandung Institute of Technology, Indonesia. She received her M.Eng degree in Electical Engineering of Telecommunication from National University of Malaysia in 2011. Her research interests include data networking, data communication, vehicular ad-hoc network and network simulation.

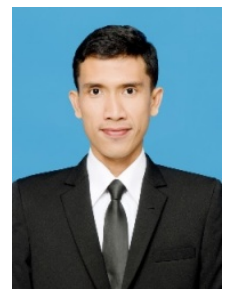

Heriansyah is a Master's Degree Student of Telecommunication Engineering at Bandung Institute of Technology, Indonesia. He has received his Bachelor of Technology degree in Electrical Engineering from Lampung University, Indonesia in 2011. His research interests include vehicular ad-hoc network, computer network and data communication. 